\title{
El mercado de trabajo en la zona suroeste metropolitana madrileña. Un ejemplo en el estudio del municipio de Fuenlabrada
}

José Murillo Castillejo

\section{INTRODUCCIÓN}

El empleo y el paro, dimensiones básicas del mercado de trabajo, constituyen actualmente la mayor preocupación en los países más desarrollados. La crisis económica mundial de los años noventa tiene también su reflejo en la región madrileña, donde sorprende la rapidez con la que está destruyendo empleo y se está produciendo el aumento del paro.

En este artículo hacemos una breve caracterización de la oferta y de la demanda de empleo, en un espacio con una alta potencialidad de paro, como es la zona suroeste metropolitana madrileña, tal como apunta la propia dinámica de su estructura demográfica. En este espacio, Fuenlabrada es uno de los principales núcleos demográficos. Es la industria, instalada a lo largo de su término municipal y que se extiende también por los municipios vecinos, el núcleo principal de la oferta de empleo existente en la zona.

El estudio se encuadra dentro de una tesis doctoral, en elaboración, cuyo objetivo es el análisis del espacio social y del empleo y su relación con el "espacio del paro" en la localidad. Para este fin se ha elaborado entre otros análisis una encuesta sobre el empleo industrial que aquí se genera. Se han encuestado medianas y grandes empresas de esta zona, recogiéndose aquí algunas de las conclusiones obtenidas.

El empleo al que nos referimos es un empleo de base contractual, que se inserta en el marco legal que señala la legislación laboral española. No se ha estudiado el empleo generado por la economía irregular o sub- 
terránea, que sabemos que también existe, pero que es difícil de cuantificar y valorar por la metodología empleada.

Se ha tratado de caracterizar el empleo no en relación a su dimensión sectorial sino a su dimensión global. Destacamos por su significación, las siguientes variables en relación al empleo industrial generado en las zonas industriales de Fuenlabrada: el empleo por sexo, por cualificación profesional, las modalidades de contratación de los trabajadores y el lugar de residencia de los mismos. Previamente vamos a localizar y clasificar la zona suroeste metropolitana, viendo sus orígenes, desarrollo y problemática, analizando también la actividad, ocupación y desempleo.

\section{LA ZONA SUROESTE METROPOLITANA MADRILEÑA}

\subsection{Localización y clasificación}

Fuenlabrada viene a incluirse en la zona denominada Suroeste Metropolitano. Situado este espacio fuera del área metropolitana administrativa incluye a municipios dentro del área metropolitana funcional, caso de Parla, Humanes de Madrid y la misma Fuenlabrada, con otros que no como Torrejón de la Calzada. El espacio delimitado se estructura territorialmente en torno at siguiente nudo de comunicaciones: carretera Leganés-Fuenlabrada-Humanes, autovía de Toledo y comarcal Móstoles-FuenlabradaPinto, delimitación que viene recogida en diferentes estudios de la región madrileña.

Otra clasificación empleada ha sido la administrativa, que utiliza la denominación de Suroeste 1 para referirse a una serie de municipios, y tal como señalaba COPLACO en 1982, con la finalidad de “concretar los aspectos ya desarrollados en las Directrices de Planeamiento para el Area Metropolitana Institucional, en dos nuevas zonas: la zona suroeste 1, que comprende los términos municipales de Móstoles, Fuenlabrada y Parla, y la zona formada por el término municipal de Alcalá de Henares" (1982: III).

\subsection{Origenes, desarrollo y problemática}

Hasta los años sesenta nos encontramos en la zona con unos pueblos rurales, con una actividad económica volcada en el sector primario y un reducido crecimiento demográfico. Esta situación «no era sino fiel reflejo de un modelo estructural compacto, centrado aún en el municipio de Madrid, debido a la todavía baja accesibilidad de la futura periferia metro- 
politana" (ÁREA Y SISTEMA. 1990). El proceso de crecimiento de estos municipios es el resultado de un complejo proceso de difusión que afecta a buena parte de la región madrileña. Este tendrá lugar siguiendo los ejes de comunicación, en saltos sucesivos que van afectando en primer lugar a los municipios de la primera corona metropolitana y posteriormente a los de la segunda.

El crecimiento es a partir de los años setenta muy intenso, pero a la vez muy anárquico y generador de importantes desequilibrios que afectarán con posterioridad. Desde el punto de vista urbano el crecimiento desordenado de la edificación residencial ha dado lugar a déficits de equipamientos, infraestructuras y servicios, incapaces de atender esta "explosión» de la demanda. Desde un punto de vista demográfico, se ha producido un importante aumento de la población, formada por jóvenes con un nivel de renta y cualificación baja. $Y$ desde el punto de vista productivo, la dispersión de la industria en sus términos municipales ha impactado negativamente en el medio natural y en los recursos agrarios. A todo ello hay que unir el desequilibrio población-empleo, agravado aún más en el sector servicios por la falta de actividades terciarias en estos núcleos, con el consiguiente impacto en la congestión del viario metropolitano de acceso a la capital.

\subsection{Actividad y ocupación}

La población activa es fundamentalmente masculina, con tasas mayores que la media regional, mientras que la tasa de actividad femenina es muy inferior a la misma. En relación a los grupos de edad se aprecian significativas diferencias. Entre los hombres la tasa crece hasta los veintinueve años, se mantiene entre los treinta y los cincuenta y cinco, para caer finalmente a partir de esta edad. La mujer sin embargo presenta la más alta tasa de actividad entre las más jóvenes, entre dieciséis y veinticuatro años, para descender progresivamente con la edad.

Las mayores tasas de actividad femenina corresponden a situaciones que se miden en posibilidades, oportunidades y perspectivas de encontrar trabajo. Las jóvenes en España cada vez tienden más a integrarse en el mundo laboral. Tal como señala Carmen de Miguel, «la tasa de actividad femenina viene creciendo de forma ininterrumpida desde el comienzo de los años ochenta, como consecuencia del fuerte aumento del nivel de instrucción femenino, los bajos niveles de fecundidad e incremento de la rotación laboral derivado de la mayor flexibilidad en la contratación" (DE MigueL, C. 1987: 21). 
En cuanto a la distribución sectorial de la población ocupada en la zona suroeste metropolitana, ésta se caracteriza por el gran peso del sector servicios, lo que contrasta con la escasa plasmación física que tiene éste en toda el área. Es el suroeste metropolitano de la Comunidad de Madrid el espacio que presenta un mayor déficit. En la década de los setenta y ochenta los municipios de esta zona, y especialmente Fuenlabrada, experimentan un gran crecimiento demográfico, auge que sin embargo no se ve acompañado de un correspondiente desarrollo del sector terciario. El segundo sector por ocupación es el industrial, ocupando la construcción una tercera posición.

\subsection{Ocupación y desempleo}

Se advierte desde los años setenta un progresivo aumento de la población ocupada por la llegada de contingentes de fuera, mayoritariamente procedentes de Madrid y núcleos de la primera corona metropolitana, que presentan una tasa de actividad superior a la media regional, pues los que se trasladan son en su mayoría personas jóvenes con empleo.

Este aumento de la población ocupada ha ido acompañado a su vez por un aumento progresivo del número de desempleados. Hay que tener en cuenta que cuando comenzaban estos municipios su crecimiento demográfico el desempleo era casi inexistente. Sin embargo en 1981 la tasa de paro era ya tres puntos superior a la media regional y nacional. En 1986 y en 1991 aún sigue siendo más elevada, aunque en este último año ya se advierte un significativo descenso del paro como resultado del fuerte crecimiento económico de estos años. El descenso se interrumpe en el segundo semestre de 1991, con un aumento progresivo del número de parados a partir de entonces.

Otra nota característica de la zona suroeste metropolitana es la fuerte precarización del empleo. La proporción de trabajadores fijos sobre el total de ocupados es relativamente baja, y aparece en relación con una mayor presencia de trabajadores con contrato temporal.

\section{LA DEMANDA DE EMPLEO EN FUENLABRADA}

\subsection{Una población muy joven y poco cualificada}

Fuenlabrada presenta una estructura demográfica cuyo hecho más significativo es su juventud. En 1991 la población de menos de quince años suponía un $34,28 \%$ en Fuenlabrada, por un $19 \%$ en la Comunidad de 
Madrid. Al mismo tiempo la población mayor de 65 años era del 2,77\%, muy baja con respecto a la media regional $(11,84 \%$ en la Comunidad de Madrid). Esta juventud de la población, unido a un mayor número de nacimientos y menor de defunciones, determina una elevada tasa de crecimiento. El crecimiento vegetativo se traduce a su vez en un importante número de niños menores de cinco años.

La consecuencia de una población tan joven es una población activa en crecimiento. Ello es resultado de la fuerte migración que llevó a Fuenlabrada matrimonios jóvenes en búsqueda de una vivienda barata en los años de la pasada crisis económica, desde mediados de los años setenta a mediados de los años ochenta. Son precisamente sus hijos quienes presionan sobre el mercado de trabajo en los años noventa, con la potencialidad de desempleo que ello supone.

Finalmente la población de Fuenlabrada se caracteriza por una baja cualificación profesional que es el resultado de un nivel de instrucción bajo. Si la proporción de analfabetos no es significativa, lo que se comprende por el elevado número de jóvenes que han recibido la educación básica obligatoria, la proporción de titulados universitarios sí lo es. Esta situación puede consolidarse al observar la baja tasa de escolarización de los mayores de 14 años, final de la etapa obligatoria, siempre menores que las medias regionales.

\subsection{Población activa e inactiva}

Si observamos la pirámide demográfica en la que aparece la distribución de ocupados, activos y parados (fig.1), vemos como hay una población activa (ocupada y parada) en mayor proporción masculina que femenina, siendo mayoritaria la primera a partir de los veintiséis años de edad. La población activa femenina sólo es equiparable con la masculina entre los 16 y 20 años. Este mayor equilibrio se produce por la base de la pirámide, al igual que también ocurre con el número de parados. La población inactiva en Fuenlabrada está formada por amas de casa, pensionistas y jubilados, y estudiantes mayores de dieciséis años.

En el grupo de inactivas, las amas de casa constituyen el grupo fundamental que explica la alta tasa de inactividad (alrededor del $20 \%$ del total de la población del municipio). En el conjunto de la población femenina mayor de 16 años y económicamente no activa supone casi el $90 \%$ de la misma. Esta proporción aumenta considerablemente en relación a la generación a la que pertenecen, a mayor edad mayor es la proporción. Hay que tener también en cuenta que el matrimonio supone en 


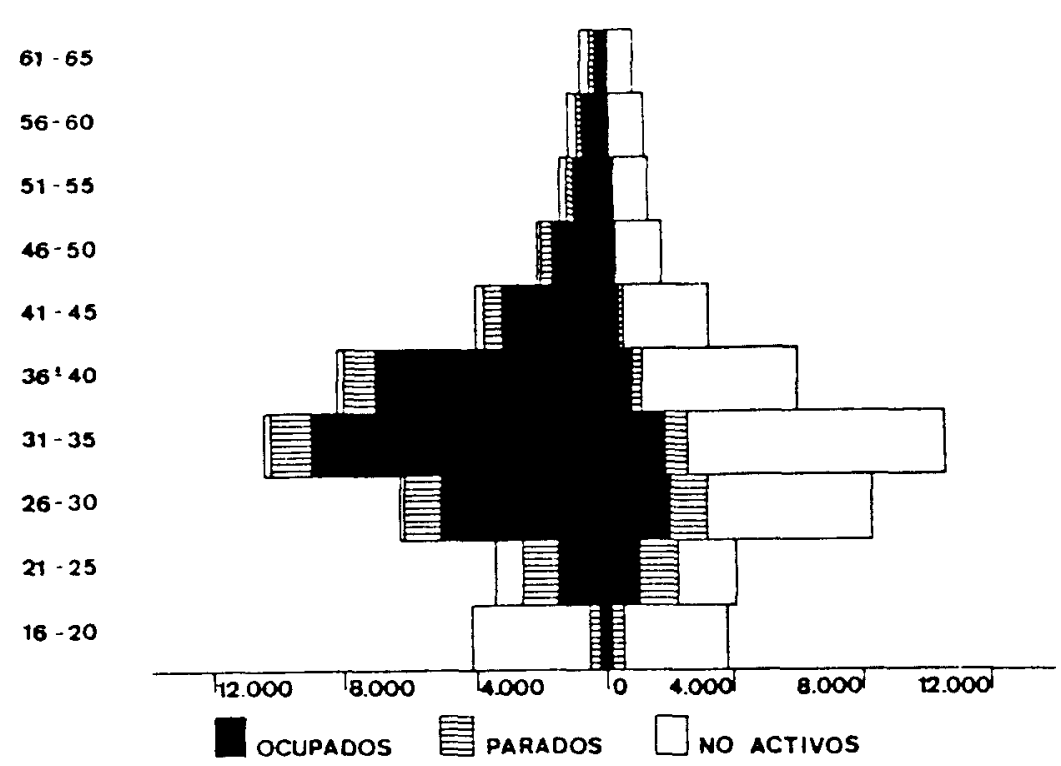

Fig. 1. Pirámide demográfica. 31-12-1988. Fuenlabrada.

situaciones de crisis económica un importante factor de disuasión para la búsqueda de empleo. En Fuenlabrada la tasa de actividad de las solteras es mayor que la de las casadas. Muchas de éstas no encontrando trabajo pasan a engrosar las filas del desempleo en mayor proporción que los varones.

\subsection{Distribución sectorial de la población ocupada}

Ésta refleja en Fuenlabrada el peso importante, en relación a las medias regionales, del empleo en la industria y en la construcción (alrededor de un $30 \%$ y un $10 \%$ respectivamente). Sin embargo es el empleo en el sector servicios el mayoritario, ya que supone un $57,4 \%$ de los ocupados residentes en Fuenlabrada. Un sector terciario de ámbito en buena parte no local, y cuya consecuencia más significativa es el desequilibrio empleoresidencia, que determina que muchos de los que aquí viven se tengan que desplazar a trabajar a la capital o a otras zonas del espacio metropolitano madrileño. 
FIGURA 2. EMPRESAS Y EMPLEO EN RELACIÓN AL VOLUMEN DE TRABAJADORES POR ESTABLECIMIENTO

\begin{tabular}{lrrrr}
\hline \multicolumn{1}{c}{ INTERVALO O EMPLEO } & ESTABLEC. $\%$ TOTAL & EMPLEO & $\%$ TOTAL \\
\hline De 0 a 2 & 113 & $12,19 \%$ & 181 & $1,33 \%$ \\
De 3 a 19 & 635 & $68,50 \%$ & 5.519 & $40,49 \%$ \\
De 20 a 49 & 143 & $15,43 \%$ & 4.362 & $32,00 \%$ \\
De 50 a 99 & 27 & $2,91 \%$ & 1.776 & $13,03 \%$ \\
De 100 a 249 & 7 & $0,76 \%$ & 1.052 & $7,72 \%$ \\
De 250 a 499 & 2 & $0,22 \%$ & 741 & $5,44 \%$ \\
\hline \multicolumn{1}{c}{ TOTAL } & 927 & $100,00 \%$ & 13.631 & $100,00 \%$ \\
\hline
\end{tabular}

Fuente: Directorio de Establecimientos Industriales. CAM., 1989.

\section{LA OFERTA DE EMPLEO EN FUENLABRADA: LA INDUSTRIA, PRINCIPAL FUENTE DE EMPLEO LOCAL}

\subsection{Caracterización del empleo industrial}

En 1989 y según datos del Directorio de Establecimientos Industriales de la Comunidad de Madrid, los empleos industriales en Fuenlabrada son 13.631, repartidos en 932 establecimientos, más de un $50 \%$ del empleo generado en toda la localidad. Recogemos en un cuadro estadístico las empresas y el empleo en relación al volumen de trabajadores por establecimiento (fig. 2).

Visto el cuadro podemos destacar algunos de los índices obtenidos. Si relacionamos el volumen de establecimientos y empleo, obtenemos una media de 14,7 empleos por establecimiento (16,64 media regional), viéndose que ésta es variable en los diferentes intervalos definidos. En las empresas de uno a diecinueve trabajadores la media es de 7,62 empleos por establecimiento (4,71 media regional), mientras que en las de más de diecinueve empleados la media es de 44,30 empleos por establecimiento (99,13 media regional). En cuanto al tamaño de los establecimientos en relación al volumen de empleo, más del $80 \%$ de los mismos se encuentran dentro del intervalo comprendido entre uno y diecinueve trabajadores, mientras que el restante $20 \%$ supera los veinte empleados por establecimiento. Vemos así como los pequeños y medianos establecimientos industriales son los más numerosos en este tejido industrial. El empleo en establecimientos con menos de cincuenta trabajadores supone casi tres cuartas partes del empleo que aquí se produce. 


\subsection{El empleo por sexo y cualificación profesional}

Sobre la base de nuestra encuesta industrial hemos comprobado que éste es un empleo básicamente masculino. El $85 \%$ del empleo total es ocupado por varones, frente a un $15 \%$ ocupado por mujeres. En todas las empresas encuestadas había trabajadoras, aunque variando su número en relación a los diferentes sectores de actividad. Sólo en las artes gráficas y la industria de la alimentación, entre los cinco sectores más representativos de la industria de Fuenlabrada (por este orden: transformados metálicos, madera, artes gráficas, plásticos y alimentación), es representativo el trabajo de la mujer en las tareas directamente productivas.

La distribución por profesiones del empleo femenino la comprobamos mediante la observación de los lugares de trabajo, tanto fábrica propiamente dicha como oficina, así como por los comentarios apuntados por los entrevistados. De todo ello se desprende que la mujer trabaja mayoritariamente en la oficina, y en la fábrica sólo en algunos sectores y por lo general con una escasa cualificación profesional. La mujer desarrolla principalmente labores de administración (recepcionistas, secretarias, y administrativas).

Con respecto a la cualificación laboral del empleo existente podemos concretar su distribución en relación a las cuatro grandes categorías profesionales delimitadas en la encuesta (directivos y técnicos, administrativos, obreros cualificados y sin cualificar). Las proporciones obtenidas en la encuesta son las siguientes: $7,4 \%$ del empleo corresponde a directivos y técnicos, $16,5 \%$ a personal administrativo y comerciales, $41,6 \%$ a oficiales y $34,5 \%$ a peonaje.

Destaca en primer término el bajo porcentaje de mano de obra empleada en labores de gestión y organización, no llegándose a una cuarta parte del total de los empleos. Podemos ver que prima el empleo directamente productivo sobre el de administración y gestión en este tejido industrial. Sin embargo esta desproporción es menor conforme aumenta el número de trabajadores de la empresa.

\subsection{La contratación laboral}

La contratación laboral viene caracterizada por un empleo fundamentalmente asalariado, que se concreta mediante la formalización de un contrato laboral entre empresario y trabajador. Equiparamos el empleo estable con la contratación de carácter fijo, y el empleo inestable con la contratación de carácter temporal. Ante un solo tipo de contrato fijo existen 
variedad de contratos temporales, estos últimos en relación con una política de fomento del empleo que España ha venido adoptando en estos últimos años, y que tuvo su origen en las altas tasas de paro generadas por la anterior crisis económica.

Hemos preguntado sobre la importancia de uno y otro tipo de contratación, y en relación a los resultados de nuestra encuesta destacamos el predominio de la contratación fija sobre la temporal. Sin embargo esto no se produce de igual modo en relación a las diferentes categorías profesionales. El empleo con carácter fijo es más importante cuanto mayor es la cualificación profesional, reduciéndose en favor del temporal a medida que descendemos. La contratación fija es dominante y por este orden, en directivos y técnicos, administrativos y obreros cualificados. La contratación temporal sólo es mayoritaria entre los obreros no cualificados.

La contratación con carácter temporal o de duración determinada surge muchas veces de las necesidades de las empresas. La legislación española la contempla cuando recoge una variedad de contratos de carácter temporal. Entre los más utilizados, el contrato por obra o servicio, el de interinidad y el de lanzamiento de una nueva actividad. A éstos, con carácter general, habría que sumar los más utilizados en la contratación de desempleados y jóvenes trabajadores, alguno de éstos con bonificaciones a la Seguridad Social.

La importancia de la contratación fija y temporal viene afectada por la edad de los trabajadores. Son los jóvenes los principales afectados por la contratación temporal. Hemos preguntado en nuestra encuesta por los tipos de contratos más habituales que se realizan a trabajadores entre los dieciséis y veinticinco años de edad. Las respuestas obtenidas señalan que éstos son por orden de importancia los siguientes: de fomento del empleo, de formación, en prácticas, a tiempo parcial, y fijo. Los contratos de carácter temporal suponen alrededor del $90 \%$ de los mismos, siendo los restantes fijos. Recordemos que la duración mínima del contrato de fomento del empleo, el más utilizado, es de seis meses (actualmente de un año tras el último cambio en la legislación) y la máxima de tres años.

En relación a la importancia de la contratación temporal, se pensó en concretar las ventajas e inconvenientes que suponía ésta para los empresarios de la zona. Ya sabemos de la dificultad que presenta la inserción de los jóvenes en el mundo laboral por la inadecuación entre el sistema educativo y las necesidades del mundo productivo, asi como de la falta de experiencia laboral anterior y de la competencia con trabajadores más experimentados. La mayoría de los encuestados, en consonancia con los contratos utilizados, destacaron las ventajas sobre los inconvenientes. Las 
razones esgrimidas fueron muy variadas, siendo la más repetida la que menciona la formación.

Para los empresarios un joven sin experiencia laboral requiere de un período de aprendizaje, y es la contratación temporal a través de sus modalidades específicas, la que permite tenerla sin costes excesivos para las empresas. Este período de prueba también permite comprobar la adaptabilidad y disposición del joven hacia el trabajo propuesto. Para muchos de los encuestados es necesario potenciar la figura del aprendiz en las empresas. Los actuales planteamientos de la reforma del mercado de trabajo van en esta dirección (crear un nuevo contrato de aprendizaje, suspender los de carácter temporal y potenciar el contrato de tiempo parcial). Otros argumentos apuntados, en favor de la contratación temporal de los jóvenes, son la reducción de costes y la necesidad de ajustar los trabajadores a las puntas de la producción. Los inconvenientes señalados vienen en relación a las limitaciones establecidas en la redacción de estos contratos.

En resumen, podemos ver como en la contratación de carácter temporal priman las reglas del mercado de trabajo irregular o externo, caracterizado por la flexibilidad laboral, salarios en relación a la oferta y la demanda y alta rotación en el empleo. Características que afectan como hemos podido ver a los trabajadores más jóvenes y a los que tienen una baja cualificación profesional. Mientras que en la contratación fija dominan las reglas del mercado regular o interno, caracterizado por la asignación y determinación del empleo. La coexistencia dentro de la empresa de los dos tipos de empleo, estable e inestable, aparece en relación con el sexo, la edad y la categoría profesional del trabajador. Esta marcada diferencia entre uno y otro tipo de empleo también tiene su importancia en otros aspectos, como por ejemplo la asignación del salario.

\subsection{Lugar de residencia de los trabajadores en las zonas industriales de Fuenlabrada}

Se pidió a los empresarios o directivos encuestados que indicasen si los empleados, trabajadores de las zonas industriales de Fuenlabrada, residen o no mayoritariamente en esta localidad. Se establece en la pregunta la diferenciación de categorías laborales al efecto. En caso negativo se solicita el lugar de residencia principal, Madrid capital u otros municipios metropolitanos.

Los resultados obtenidos señalan que un $38,2 \%$ de los mismos reside en la localidad, un $46 \%$ en los municipios limítrofes y el restante $15,8 \%$ 
en Madrid capital. La residencia de los trabajadores tiene una significativa relación con la distancia al poligono industrial en el que se trabaja, aunque no pueda hablarse de correlación. Ello ha podido comprobarse al diferenciar a los trabajadores en relación a las cuatro grandes zonas industriales en las que se localizan los polígonos de la localidad (fig. 3).

La Zona Oeste, zona industrial situada al oeste de nuestro término municipal, tiene una importante vinculación con los pueblos de este área, Móstoles y Leganés fundamentalmente. En la Zona Este, situada junto a la carretera de Toledo, la vinculación se produce en cambio con municipios como Getafe, Parla y Pinto. En las otras dos zonas industriales (Zona Estación y Centro), éstas más en el entorno del casco urbano, el empleo local es mayoritario.

Finalmente hemos señalado el lugar de residencia en relación a la categoría profesional. Los empleos más cualificados, empresarios, directivos y técnicos, viven fundamentalmente en Madrid. El personal administrativo se reparte entre Fuenlabrada, los municipios limítrofes y Madrid. Los obreros cualificados residen sobre todo en los municipios vecinos de Fuenlabrada (Getafe, Móstoles, Humanes, Parla, Leganés y Pinto), seguido del propio Fuenlabrada y de Madrid, aunque este último con escasa representación. $Y$ finalmente los obreros no cualificados, que residen principalmente en el lugar más cercano, primeramente en Fuenlabrada y después en los municipios próximos. La residencia en la capital en este caso es prácticamente nula.

\section{CONCLUSIONES}

Hemos concretado la demanda y la oferta de empleo en Fuenlabrada, destacando una serie de factores que explican también el mercado de trabajo en la zona suroeste metropolitana, pues los municipios integrantes de este área son pueblos con parecida génesis y problemática.

Entre los factores de la demanda hay que destacar el ciclo demográfico, la evolución de la propia estructura demográfica, que viene repercutiendo en una mayor demanda de trabajo en los años noventa. Ello traerá como resultado un aumento de la potencialidad de desempleo, con una incidencia menor o mayor en función de la generación o no de empleo en toda la región madrileña, lo que depende en gran medida de la coyuntura o ciclo económico. Otro importante factor es el nivel de estudios y la formación, que tienen una clara repercusión sobre las posibilidades de encontrar empleo.

Por lo que se refiere a la oferta de empleo industrial generado en la zona hemos apuntado una clara división social del trabajo entre los sexos, 


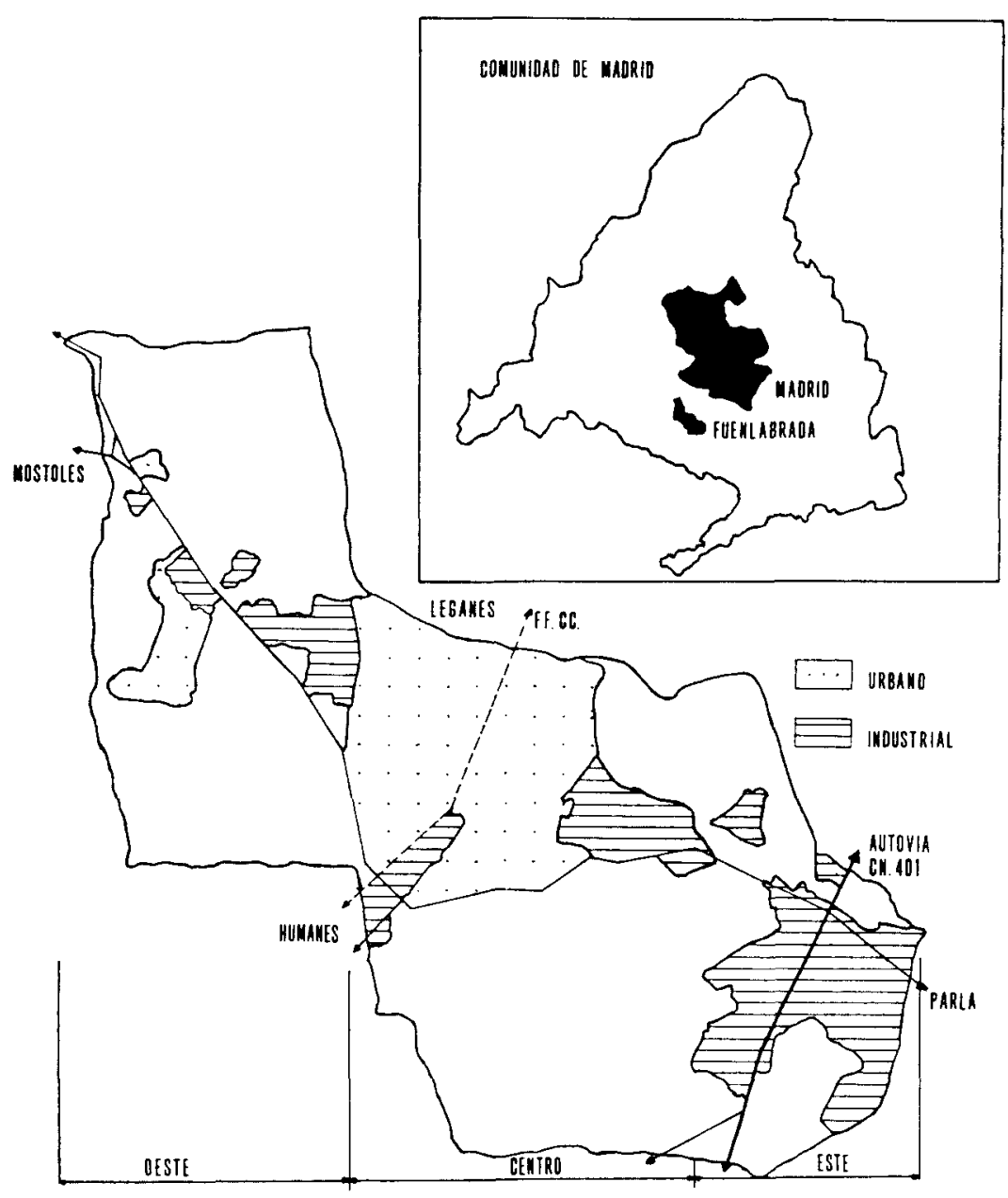

FIGURA-3. NUCLEOS URBAHOS Y ZOMAS INOUSTRIALES. FuERLARRABa ESC 1: 75.000

Fig. 3. Núcleos urbanos y zonas industriales. Fuenlabrada Esc. 1:75.000. 
la importancia de los contratos temporales en relación al empleo de los jóvenes trabajadores y la fuerte movilidad laboral de los trabajadores de las zonas industriales de Fuenlabrada.

Parece evidente que la oferta existente de empleo no va a reducir el paro en la zona, pues es una oferta ya saturada y estancada con la nueva crisis económica. A ello habría que sumar la desindustrialización creciente en sectores de fuerte implantación en la zona, en gran medida por la competencia de otros espacios periféricos, más alejados y ya fuera de la propia Comunidad de Madrid, que están ofreciendo mayores ventajas para su localización.

Las oportunidades de empleo, y sobre todo para los jóvenes, pasan por una elevación del nivel de formación y cualificación profesional, 10 que les permitiría acceder al nuevo empleo que continuamente el mercado de trabajo, en constante transformación por las nuevas tecnologías, está demandando.

\section{BIBLIOGRAFIA}

Agüero, 1. y Olano Rey, A. 1988: “Oferta de trabajo de jóvenes: aspectos demográficos y económicos". Revista de Economía y Sociología del Trabajo, n/ 1 y 2. Madrid, págs. 12-29.

Anorés, J. y García, J. 1991: “El nivel de estudios como factor explicativo del desempleo, de los ingresos y de la movilidad industrial». Economia Industrial, n. ${ }^{\circ} 278$. Madrid, págs. 13-22.

ÁREA y SistemA. 1986: “Areas de problemática laboral homogénea en el territorio de la Comunidad de Madrid». Alfoz, núm. 45. Madrid, págs. 30-49.

- 1990: El distrito industrial de la periferia metropolitana del suroeste. Madrid, Consejeria de Economía de la Comunidad de Madrid.

CELADA, F. 1988: “El distrito industrial de la periferia suroeste del área metropolitana de Madrid». Economia y Sociedad, n. ${ }^{\circ}$. Madrid, págs. 70-85.

Comunidad de Madrid. 1992: Boletín de Coyuntura $4 .^{\circ}$ trimestre $1.992, n .{ }^{\circ} 34$. Madrid, Consejeria de Economía, 164 páginas.

- 1989: Directorio de establecimientos industriales de la CAM. Madrid, Consejería de Economía, 133 páginas.

- 1988: «Informe: una estrategia para la zona sur metropolitana». Alfoz, $n .^{\circ} 56$. Madrid, págs. 84-146.

COPlaco. 1982: Directrices de planeamiento territorial urbanístico para la revisión de los planes generales de los términos municipales de Alcalá de Henares, Fuenlabrada, Móstoles y Parla. Madrid, MOPU, 177 páginas.

De Miguel, C. y Agüero,I. 1986: “Evolución demográfica y oferta de fuerza de trabajo", Tendencias Demográficas y Planificación Económica. Madrid, Ministerio de Economía y Hacienda.

Estébanez, J. (edit.) 1990: Madrid, presente y futuro. Madrid, Akal. 
MARCos, C. y SANz, L. 1988: “Tendencias recientes en el mercado de trabajo madrileño", Alfoz, núm. 47, págs 21-34.

MÉNDEZ, R. (1986): Actividad industrial y estructura territorial en la región de Madrid. Consejeria de Industria, Trabajo y Comercio de la Comunidad de Madrid, 335 páginas.

Santos Preciado, J.M. 1988: El modelo de diferenciación residencial del sector suroeste del Area Metropolitana de Madrid. Madrid.

\section{RESUMEN}

Hacemos en este artículo una breve caracterización del mercado de trabajo en uno de los municipios metropolitanos madrileños, Fuenlabrada, principal fuente de empleo industrial de la zona suroeste de la Comunidad de Madrid. La localidad se encuentra afectada por una tasa de paro por encima de la media regional, siendo su potencialidad de desempleo alta en relación a su estructura demográfica, elevado número de jóvenes con tendencia a aumentar, y a un bajo nivel educativo de la población residente. Sobre estas premisas caracterizamos el empleo industrial generado en los polígonos de Fuenlabrada, y en relación a las variables siguientes: sexo, cualificación profesional, modalidades de contratación y lugar de residencia de la población que aquí trabaja.

\section{ABSTRACT}

The aim of this paper is to show a short profile about the labor market in Fuenlabrada, a Metropolitan Community of Madrid and one of the main sources of industrial employment. Fuenlabrada has an unemployment rate higher than the regional average value and a potential unemployment rate very high, according its demographic structure, a great deal of growing young people and a very poor educational level. On these premises we analyse the industrial employment generated by the industrial estates located in Fuenlabrada. We relate too the creation of employment with some variables such as sex composition, professional level and place of residence of the population working in Fuenlabrada. 